\title{
THE DEHN SURGERY CHARACTERIZATION OF THE TREFOIL AND THE FIGURE EIGHT KNOT
}

\author{
PETER OZSVÁTH AND ZOLTÁN SZABÓ
}

\begin{abstract}
We give a Dehn surgery characterization of the trefoil and the figure eight knots. These results are gotten by combining surgery formulas in Heegaard Floer homology from an earlier paper with the characterization of these knots in terms of their knot Floer homology given in a recent paper of Ghiggini.
\end{abstract}

\section{INTRODUCTION}

In [3], it was shown that the unknot is characterized by its Dehn surgeries, in the sense that if Dehn surgery with some slope on a knot $K$ in $S^{3}$ is orientation-preserving homeomorphic to Dehn surgery with the same slope on the unknot, then $K$ is in fact unknotted.

A proof of this fact can be given using Heegaard Floer homology [9]. Specifically, there is a Heegaard Floer homology theory for knots introduced in [8] and [12]. Surgery formulas for this invariant [10] allow one to express the Heegaard Floer homology for $p / q$-Dehn surgery of $K$ in terms of this knot Floer homology of $K$. The hypothesis that $p / q$ surgery on $K$ agrees with that of the unknot forces the knot Floer homology of $K$ to agree with that of the unknot. Combining this with the fact that knot Floer homology detects the unknot [7], the Dehn surgery characterization of the unknot follows.

In a beautiful recent paper, Ghiggini [2] shows that Heegaard Floer homology also detects the trefoil and the figure eight knot. Appealing to the same strategy outlined above, in the form of the surgery formulas for knot Floer homology, we obtain here a similar Dehn surgery characterization of both of these knots. Specifically, we have the following:

Theorem 1.1. Let $T$ be a trefoil knot. If $K$ is a knot with the property that there is a rational number $r$ and an orientation-preserving diffeomorphism $S_{r}^{3}(K) \cong S_{r}^{3}(T)$, then $K$ is in fact the trefoil $T$.

In a similar vein, we have the following

Key words and phrases. Floer homology, trefoil knot, figure eight knot, Dehn surgery.

PSO was supported by NSF grant number DMS-050581.

ZSz was supported by NSF grant number DMS-0406155. 
Theorem 1.2. Let $S$ be the figure eight knot. If $K$ is a knot with the property that there is a rational number $r$ and an orientation-preserving diffeomorphism $S_{r}^{3}(K) \cong S_{r}^{3}(S)$, then $K$ is in fact the figure eight knot $S$.

The condition that the diffeomorphism preserves orientations is important, here. For example, there are identifications $S_{+1}^{3}\left(T_{\ell}\right) \cong-S_{+1}^{3}(S)$, and also $S_{+5}^{3}\left(T_{r}\right) \cong-S_{+5}^{3}(O)$, where here $O$ is the unknot.

Similarly, the condition that the surgery coefficient is fixed is also crucial; $S_{1 / n}^{3}\left(K_{0}\right)$ where $K_{0}$ is a trefoil or the figure eight knot can be realized alternatively as +1 surgery on a suitable twist knot.

In his paper, Ghiggini proves that the trefoil is the only knot in $S^{3}$ which admits a surgery giving the Poincaré homology sphere. A consequence of Theorems 1.1 and 1.2, we obtain a similar result for the Brieskorn sphere $\Sigma(2,3,7)$.

Corollary 1.3. The only surgeries on knots in $S^{3}$ which realize the Brieskorn sphere $\Sigma(2,3,7)$ (with either orientation) are $S_{-1}^{3}\left(T_{r}\right) \cong S_{+1}^{3}(S) \cong \Sigma(2,3,7)$ and $S_{+1}^{3}\left(T_{\ell}\right) \cong$ $S_{-1}^{3}(S) \cong-\Sigma(2,3,7)$.

In Section 2, we review the relevant aspects of Heegaard Floer homology which are used in the proofs of the above results. In Section 3 we give the proofs of the above two theorems and the corollary. 


\section{BACKGROUND}

2.1. Heegaard Floer homology. In its most basic form, Heegaard Floer homology is a $\mathbb{Z} / 2 \mathbb{Z}$-graded Abelian group associated to a three-manifold, but it comes in several variants and can be endowed with additional structure [9].

In this paper, we will consider primarily the version $H^{+}(Y)$ for rational homology three-spheres $Y$. This group admits a splitting according to $\operatorname{Spin}^{c}$ structures over $Y$

$$
H F^{+}(Y) \cong \bigoplus_{\mathfrak{t} \in \operatorname{Spin}^{c}(Y)} H F^{+}(Y, \mathfrak{t}) \text {. }
$$

Moreover, $\mathrm{HF}^{+}(Y, \mathfrak{t})$ is equipped with an absolute grading (defined in [4, Section 7] and studied extensively in [5]). Recall that there is a natural involution on the space of $\operatorname{Spin}^{c}$ structures over $Y$, denoted $\mathfrak{t} \mapsto \overline{\mathfrak{t}}$. There is a corresponding isomorphism

$$
H F^{+}(Y, \mathfrak{t}) \cong H F^{+}(Y, \overline{\mathfrak{t}})
$$

which, in the case of rational homology spheres $Y$, is an isomorphism of $\mathbb{Q}$-graded Abelian groups.

The group $H F^{+}(Y, \mathfrak{t})$ has the following algebraic structure.

$$
H F^{+}(Y, \mathfrak{t})=\bigoplus_{d \in \mathbb{Q}} H F^{+}(Y, \mathfrak{t})
$$

In fact, $\mathrm{HF}^{+}(Y, \mathfrak{t})$ is supported only in rational degrees $d$ within some fixed equivalence class (depending on $Y$ and $\mathfrak{t}$ ) modulo the integers. For any degree $d \in \mathbb{Q}, H F_{d}^{+}(Y, \mathfrak{t})$ is a finitely generated $\mathbb{Z}$-module. Moreover, $\operatorname{HF}^{+}(Y, \mathfrak{t})$ is endowed with an endomorphism $U$ which lowers degree by 2 , i.e.

$$
U: H F_{d}^{+}(Y, \mathfrak{t}) \longrightarrow H F_{d-2}^{+}(Y, \mathfrak{t}) .
$$

Moreover, $H F_{d}^{+}(Y, \mathfrak{t})=0$ for all sufficiently small $d$. Finally, for any sufficiently large rational number $d_{0}$, if we consider the quotient module $H F_{\geq d_{0}}^{+}(Y, \mathfrak{t})$ of $H^{+}(Y, \mathfrak{t})$ generated by all elements with degree greater than $d_{0}$, then that module is isomorphic to the $\mathbb{Z}[U]$-module

$$
\mathcal{T}^{+}=\frac{\mathbb{Z}\left[U, U^{-1}\right]}{U \cdot \mathbb{Z}[U]} .
$$

From the above properties, it is clear that there is a canonical short exact sequence

$$
0 \longrightarrow \mathcal{T}^{+} \longrightarrow \mathrm{HF}^{+}(Y, \mathfrak{t}) \longrightarrow \mathrm{HF}_{\text {red }}^{+}(Y, \mathfrak{t}) \longrightarrow 0,
$$

where here $H F_{\text {red }}^{+}(Y, \mathfrak{t})$ is $\mathbb{Z}[U]$ module which is a finitely generated $\mathbb{Z}$-module. Moreover, we obtain a three-manifold invariant,

$$
d: \operatorname{Spin}^{c}(Y) \longrightarrow \mathbb{Q},
$$

the correction terms of $Y$, where $d(Y, \mathfrak{t})$ is the minimal $\mathbb{Q}$-grading of any homogeneous element of $H^{+}(Y, \mathfrak{t})$ in the image of $\mathcal{T}^{+}$. 
The Floer homology group $H F^{+}(Y, \mathfrak{t})$ also inherits a $\mathbb{Z} / 2 \mathbb{Z}$-grading; a non-zero element in $H F_{d}^{+}(Y, \mathfrak{t})$ has even parity if $d \equiv d(Y, \mathfrak{t})(\bmod 2 \mathbb{Z})$, and it has odd parity if $d \equiv d(Y, \mathfrak{t})+1(\bmod 2 \mathbb{Z})$. The correction terms are analogous to a gauge-theoretic invariant introduced by Frøyshov [1]; for more information on the correction terms, see $[5]$.

If $C$ is a chain complex of $\mathbb{Z}[U]$-modules, we can form

$$
H^{\mathrm{red}}(C)=\lim _{\overleftarrow{d}} \frac{H_{*}(C)}{U^{d} H_{*}(C)} .
$$

Then, if $C F^{+}(Y, \mathfrak{t})$ is the chain complex calculating $\mathrm{HF}^{+}(Y, \mathfrak{t})$, then $H^{\mathrm{red}}\left(C F^{+}(Y, \mathfrak{t})\right)=$ $H F_{\text {red }}^{+}(Y, \mathfrak{t})$.

2.2. Knot Floer homology and the surgery formula. Heegaard Floer homology can be extended as in [8] and [12] to invariants for null-homologous knots $K$ in closed three-manifolds. We restrict attention to the case where the ambient three-manifold is the three-sphere $S^{3}$. We recall now the notation of knot Floer homology, following [8]. This data can be used to calculate Heegaard Floer homology groups of Dehn fillings of $S^{3}$ along $K$, cf. [10]. After setting up notation for knot Floer homology, we state this surgery formula.

A $\mathbb{Z} \oplus \mathbb{Z}$-filtered chain complex is a free Abelian group which splits as a direct sum $C=\bigoplus_{(i, j) \in \mathbb{Z} \oplus Z} C\{(i, j)\}$ and which is endowed with a boundary operator which carries elements in $C\{(i, j)\}$ to elements in

$$
\bigoplus_{\left(i^{\prime}, j^{\prime}\right) \leq(i, j)} C\left\{\left(i^{\prime}, j^{\prime}\right)\right\},
$$

where we write

$$
\left(i^{\prime}, j^{\prime}\right) \leq(i, j)
$$

if $i^{\prime} \leq i$ and $j^{\prime} \leq j$. In the present paper, we will consider $\mathbb{Z} \oplus \mathbb{Z}$-filtered $\mathbb{Z}[U]$ complexes. These come equipped with a chain map isomorphism $U: C \longrightarrow C$ which carries $C\{(i, j)\}$ to $C\{(i-1, j-1)\}$.

Consider a subset $X \subset \mathbb{Z} \oplus \mathbb{Z}$ with the property that if $(i, j) \in X$, then for any $\left(i^{\prime}, j^{\prime}\right) \leq(i, j)$, we also have that $\left(i^{\prime}, j^{\prime}\right) \in X$.If $C$ is any $\mathbb{Z} \oplus \mathbb{Z}$-filtered chain complex, we can form a subcomplex $C\{X\} \subset C$ generated by $\bigoplus_{(i, j) \in X} C\{(i, j)\}$.

If $Y \subset \mathbb{Z} \oplus \mathbb{Z}$ a set with the property that for any $(i, j) \in Y$, if $\left(i^{\prime}, j^{\prime}\right) \geq(i, j)$ we have that $\left(i^{\prime}, j^{\prime}\right) \in Y$. In this case, we can endow $\bigoplus_{(i, j) \in Y} C\{(i, j)\}$ with the structure of a quotient complex, which we will also denote by $C\{Y\}$.

If $K \subset S^{3}$ is a knot, we obtain an associated $\mathbb{Z} \oplus \mathbb{Z}$-filtered chain complex $C$ with total homology isomorphic to $\mathbb{Z}\left[U, U^{-1}\right]$. The filtered chain homotopy type of this complex $C=\mathrm{CFK}^{\infty}\left(S^{3}, K\right)$ is a knot invariant, [8], [12]. 
The differential on $C$ induces also differential on each summand $C\{(i, j)\}$. The homology group $H_{*}(C\{(0, s)\})$ is called the knot Floer homology group in filtration level $s$, and it is denoted $\widehat{\operatorname{HFK}}(K, s)$.

The filtered chain homotopy type $\operatorname{CFK}^{\infty}\left(S^{3}, K\right)$ gives rise to some further algebraic structure.

Let $B^{+}=C\{i \geq 0\}$. This is a model for $C F^{+}\left(S^{3}\right)$, and indeed, so is $C\{j \geq 0\}$. There is a distinguished chain homotopy equivalence between these two chain complexes.

We have also chain complexes $A_{s}^{+}(K)=C\{\max (i, j-s) \geq 0\}$, equipped with a pair of maps

$$
v_{s}^{+}: A_{s}^{+}(K) \longrightarrow B^{+} \text {and } h_{s}^{+}: A_{s}^{+}(K) \longrightarrow B^{+}
$$

where the first is simply projection map (from $C\{\max (i, j-s) \geq 0\}$ to $C\{i \geq 0\}$ ), while the second is a composite of the projection map $C\{\max (i, j-s) \geq 0\}$ to $C\{j \geq s\}$, followed by the identification with $C\{j \geq 0\}$ (induced by multiplication by $U^{s}$ ), followed by the chain homotopy equivalence of this with $B^{+}$. These maps are the data necessary to calculate the Heegaard Floer homology of arbitrary Dehn fillings of $S^{3}$ along $K$.

Recall that the Heegaard Floer homology of $Y$ admits a direct sum splitting indexed by the set of $\operatorname{Spin}^{c}$ structures over $Y$, which in turn is an affine space for $H^{2}(Y ; \mathbb{Z})$. In particular, if $K \subset S^{3}$, then there is a splitting

$$
H F^{+}\left(S_{p / q}^{3}(K)\right) \cong \bigoplus_{i \in \mathbb{Z} / p \mathbb{Z}} H F^{+}\left(S_{p / q}^{3}(K), i\right)
$$

Fix an integer $i$, and consider the chain complexes

$$
\mathbb{A}_{i}^{+}=\bigoplus_{s \in \mathbb{Z}}\left(s, A_{\left\lfloor\frac{i+p s}{q}\right\rfloor}^{+}(K)\right) \text { and } \mathbb{B}_{i}^{+}=\bigoplus_{s \in \mathbb{Z}}\left(s, B^{+}\right),
$$

where here $\lfloor x\rfloor$ denotes the greatest integer smaller than or equal to $x$. We view the above chain homomorphisms $v^{+}$and $h^{+}$as maps

$$
v^{+}:\left(s, A_{\left\lfloor\frac{i+p s}{q}\right\rfloor}^{+}(K)\right) \longrightarrow\left(s, B^{+}\right) \text {and } h^{+}:\left(s, A_{\left\lfloor\frac{i+p s}{q}\right\rfloor}^{+}(K)\right) \longrightarrow\left(s+1, B^{+}\right) .
$$

Adding these up, we obtain a chain map

$$
D_{i, p / q}^{+}: \mathbb{A}_{i}^{+} \longrightarrow \mathbb{B}_{i}^{+}
$$

i.e.

$$
D_{i, p / q}^{+}\left\{\left(s, a_{s}\right)\right\}_{s \in \mathbb{Z}}=\left\{\left(s, b_{s}\right)\right\}_{s \in \mathbb{Z}},
$$

where here

$$
b_{s}=v_{\left\lfloor\frac{i+p s}{q}\right\rfloor}^{+}\left(a_{s}\right)+h_{\left\lfloor\frac{i+p(s-1)}{q}\right\rfloor}^{+}\left(a_{s-1}\right) .
$$

Let $\mathbb{X}_{i, p / q}^{+}$denote the mapping cone of $D_{i, p / q}^{+}$. Note that $\mathbb{X}_{i, p / q}^{+}$depends on $i$ only through its congruence class modulo $p$. Note also that $A_{s}^{+}$and $B_{s}^{+}$are relatively $\mathbb{Z}_{-}^{-}$ graded, and the homomorphisms $v_{s}^{+}$and $h_{s}^{+}$respect this relative grading. The mapping 
cone $\mathbb{X}_{i}^{+}$can be endowed with a relative grading, with the convention that $D_{i, p / q}^{+}$drops the grading by one.

The following is proved (in somewhat more generality) in Theorem 1.1 of [10]:

Theorem 2.1. Let $K \subset S^{3}$ be a knot, and let $p, q$ be a pair of relatively prime integers. Then, there is an identification $\sigma: \mathbb{Z} / p \mathbb{Z} \longrightarrow \operatorname{Spin}^{c}\left(S_{p / q}^{3}(K)\right)$ such that for each $i \in$ $\mathbb{Z} / p \mathbb{Z}$, there is a relatively graded isomorphism of groups

$$
\Phi_{K, i}: H_{*}\left(\mathbb{X}_{i, p / q}^{+}(K)\right) \stackrel{\cong}{\longrightarrow} H F^{+}\left(S_{p / q}^{3}(K), \sigma(i)\right)
$$

Indeed, there is a uniquely specified absolute grading on the subcomplex $\mathbb{B}_{i}^{+} \subset \mathbb{X}_{i, p / q}^{+}(K)$ (which is independent of $K$ ) for which the map $\Phi_{O, i}$ is an isomorphism (where here $O$ is the unknot). With the corresponding induced grading on $\mathbb{X}_{i, p / q}^{+}(K), \Phi_{K}$ becomes an absolutely graded isomorphism.

It is useful to note that there is a conjugation on knot Floer homology related to the conjugation invariance on closed manifolds, cf. Equation (1). In the form which we need it, this is an isomorphism

$$
\Psi: H_{*}\left(A_{s}^{+}\right) \stackrel{\cong}{\longrightarrow} H_{*}\left(A_{-s}^{+}\right)
$$

for all integers $s$. Indeed, under this isomorphism, we have a homotopy-commutative diagram commutative diagram

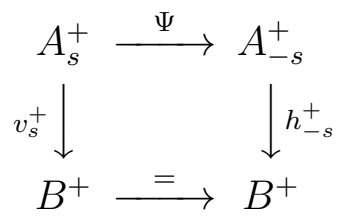

It is also useful to note that, although $\mathbb{X}_{i, p / q}^{+}(K)$ is a very large chain complex, if we are interested in the homology in degrees less than or equal to some fixed quantity $d$, then this is contained in much smaller chain complex. More precisely, suppose that $p, q>0$. Then, since $v_{s}^{+}$is an isomorphism for all sufficiently large $s$ and $h_{s}^{+}$is an isomorphism for all sufficiently small $s$, we can consider the subsets

$$
\begin{gathered}
\mathbb{A}_{i, \leq \sigma}^{+}=\bigoplus_{\{s \in \mathbb{Z}|| s \mid \leq \sigma\}}\left(s, A_{\left\lfloor\frac{i+p s}{q}\right\rfloor}^{+}(K)\right) \subset \mathbb{A}_{i}^{+} \\
\mathbb{B}_{i \leq \sigma}^{+}=\bigoplus_{\{s \in \mathbb{Z} \mid-\sigma<s \leq \sigma\}}\left(s, B^{+}\right) \subset \mathbb{B}_{i}^{+} .
\end{gathered}
$$

The map $D_{i, p / q}^{+}$induces a map from $\mathbb{A}_{i, \leq \sigma}^{+}$to $\mathbb{B}_{i, \leq \sigma}^{+}$, whose mapping cone, denoted $\mathbb{X}_{i, p / q, \leq \sigma}^{+}$, is a quotient complex of $\mathbb{X}_{i, p / q, \leq \sigma}^{+}$. Now, the homology of the chain complex $\mathbb{X}_{i, p / q}^{+}(K)$ in degrees less than or equal to $d$ agrees with the homology of its quotient complex $\mathbb{X}_{i, p / q, \leq \sigma}^{+}(K)$ for some $\sigma$ depending on $d$. 
2.3. Examples. For $K$ the unknot, $C$ has a single generator $a$ as a $Z\left[U, U^{-1}\right]$-module, which is supported in filtration level $(0,0)$ and grading zero. The differentials are trivial.

We let $T_{\ell}$ denote the left-handed trefoil, $T_{r}$ denote the right-handed trefoil, and $S$ denote the figure eight knot.

For $K=T_{r}$, we have that $C$ has three generators as a $\mathbb{Z}\left[U, U^{-1}\right]$-module, $a, b, c$, in filtration levels $(-1,0)(0,0)$, and $(0,-1)$ respectively, with the differential $D b=$ $a+c, D a=D c=0$.

For $K=T_{\ell}, C$ has three generators as a $\mathbb{Z}\left[U, U^{-1}\right]$-module, $a, b, c$, in filtration levels $(0,1)(0,0)$, and $(1,0)$ respectively, with the differential $D a=D c=b$ and $D b=0$.

Finally, for $K=S, C$ has five generators as a $\mathbb{Z}\left[U, U^{-1}\right]$ module, $a, b, c, d, e$. Here $a$ is supported in filtration level $(1,1), b$ in $(0,1), c$ in $(1,0)$, and $d$ and $e$ in $(0,0)$. Differentials are given by $D a=b+c, D b=-D c=d, D d=D e=0$.

These answers are illustrated in Figure 1.

These examples are all fairly simple to calculate; the calculations can be made by finding appropriate Heegaard diagrams, or referring to more general results from [11], $[6]$.

They have the following consequences for $A_{s}^{+}$.

Proposition 2.2. The groups $H_{*}\left(A_{s}^{+}\left(K_{0}\right)\right)$ and the homomorphisms $v_{s}^{+}$and $h_{s}^{+}$for $K=T_{r}, T_{\ell}$, and $S$ are determined as follows. For all $s \in \mathbb{Z}, H_{*}\left(A_{s}^{+}\left(T_{r}\right)\right) \cong \mathcal{T}^{+}$; indeed, for all $s>0$, the map induced by $v_{s}$ is an isomorphism, while for $s=0$, the map

$$
v_{0}: H_{*}\left(A_{0}^{+}\left(T_{r}\right)\right) \cong \mathcal{T}^{+} \longrightarrow H F^{+}\left(S^{3}\right) \cong \mathcal{T}^{+}
$$

is modeled on multiplication by $U$.

For all $s>0, H_{*}\left(A_{s}^{+}\left(T_{\ell}\right)\right) \cong \mathcal{T}^{+}$, while $H_{*}\left(A_{0}^{+}\left(T_{\ell}\right)\right) \cong \mathcal{T}_{(0)}^{+} \oplus \mathbb{Z}_{(0)}$, where the extra $\mathbb{Z}$ has grading zero. Moreover, the kernel of $v_{0}$ is one-dimensional.

For all $s>0, H_{*}\left(A_{s}^{+}\left(T_{\ell}\right)\right) \cong \mathcal{T}^{+}$, while $H_{*}\left(A_{0}^{+}\left(T_{\ell}\right)\right) \cong \mathcal{T}_{(0)}^{+} \oplus \mathbb{Z}_{(-1)}$, and the kernel of $v_{0}$ is one-dimensional.
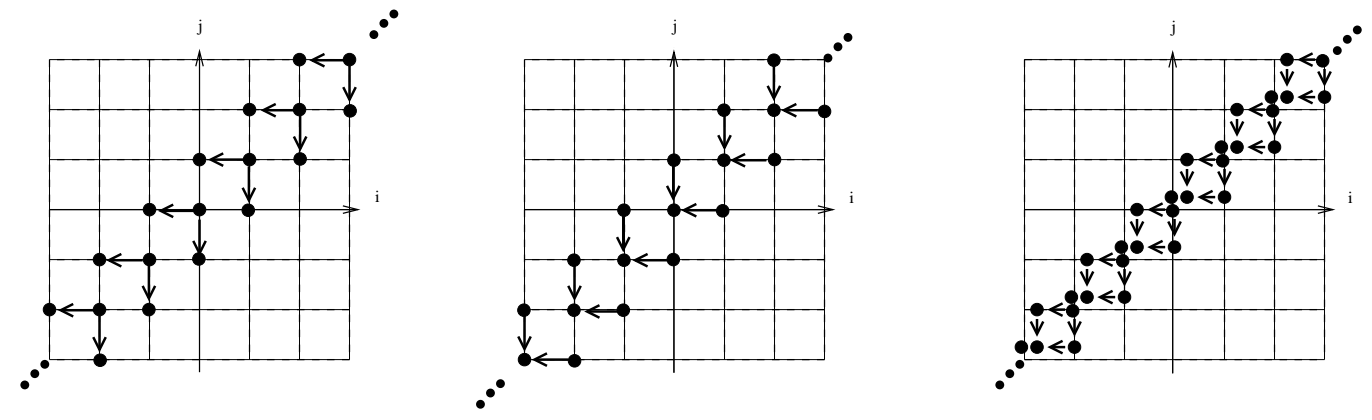

Figure 1. Filtered complexes for $T_{r}, T_{\ell}$, and $S$. We have illustrated the $\mathbb{Z} \oplus \mathbb{Z}$-filtered complexes associated to the three knots listed above. Dots represent generators, and arrows represent differentials. 
Proof. These are straightforward consequences of the chain complexes described above.

2.4. Genus bounds and Ghiggini's theorem. In [7], it is shown that if $K \subset S^{3}$ is a knot with genus $g$, then

$$
\max \left\{s \mid H_{*}(C\{(0, s)\})=\widehat{\operatorname{HFK}}(K, s) \neq 0\right\}=g .
$$

It is conjectured that if $\widehat{\operatorname{HFK}}(K, s)$ has rank one, then $K$ is a fibered knot. In a recent paper, Ghiggini verified this conjecture for knots with genus one. Taken together, these results give the following:

Theorem 2.3. Let $K$ be a knot with $\widehat{\operatorname{HFK}}(K, s)=0$ for all $s>1$ and $\widehat{\operatorname{HFK}}(K, 1)$ having rank one. Then, $K$ is a trefoil or the figure eight knot. 


\section{Proof of TheOrem 1.1 AND 1.2}

Let $K_{0}$ be a trefoil or the figure eight knot. From a graded isomorphism

$$
H F^{+}\left(S_{p / q}^{3}(K)\right) \cong H F^{+}\left(S_{p / q}^{3}\left(K_{0}\right)\right)
$$

we would like to use the surgery formula to conclude an isomorphism between the knot Floer homologies of $K$ and $K_{0}$. To this end, we find it useful to identify $H F^{+}\left(S_{p / q}^{3}\left(K_{0}\right)\right)$.

Proposition 3.1. Let $p$ and $q$ be relatively prime, positive integers. We have that

$$
\operatorname{rk} H F_{\text {red }}^{+}\left(S_{p / q}^{3}\left(T_{r}\right)\right)<q
$$

while

$$
\operatorname{rk} H F_{\text {red }}^{+}\left(S_{p / q}^{3}\left(T_{\ell}\right)\right)=\operatorname{rk} H F_{\text {red }}^{+}\left(S_{p / q}^{3}(S)\right)=q .
$$

In the case where $K_{0}=T_{\ell}, H F_{\text {red }}^{+}\left(S_{p / q}^{3}\left(T_{\ell}\right)\right)$ is supported in even degree, while in $H F^{+}\left(S_{p / q}^{3}(S)\right)$, it is supported in odd degree. Moreover, for $K_{0}=T_{r}, T_{\ell}$, and $S$, we have that

$$
\sum_{i} \operatorname{rk} H F_{\mathrm{red}}^{+}\left(S_{p / q}^{3}\left(K_{0}\right), i\right)-\left(\frac{d\left(S_{p / q}^{3}\left(K_{0}\right), i\right)-d\left(S_{p / q}^{3}(O), i\right)}{2}\right)=q .
$$

Proof. This is a straightforward application of the surgery formula (Theorem 2.1) and the calculations from Subsection 2.3.

Proposition 3.2. If $K \subset S^{3}$ is a knot with the property that for some $s>0$, the map $H_{*}\left(A_{s}^{+}(K)\right) \longrightarrow \mathrm{HF}^{+}\left(S^{3}\right)$ induced by $v^{+}$is not an isomorphism, then for any $p / q>0$, we have that

$$
\sum_{i} \operatorname{rk} H F_{\text {red }}^{+}\left(S_{p / q}^{3}(K), i\right)-\left(\frac{d\left(S_{p / q}^{3}(K), i\right)-d\left(S_{p / q}^{3}(O), i\right)}{2}\right) \geq 2 q .
$$

Proof. Suppose that $H^{\text {red }}\left(A_{s}^{+}\right) \neq 0$. Then, we can find an element of $H_{*}\left(A_{s}^{+}\right)$with non-trivial image in $H^{\mathrm{red}}\left(A_{s}^{+}\right)$which is also contained in the kernel of the map

$$
v_{*}^{+}: H_{*}\left(A_{s}^{+}\right) \longrightarrow H_{*}\left(B^{+}\right) .
$$

From symmetry, the same is true for

$$
h^{+}: H_{*}\left(A_{-s}^{+}\right) \longrightarrow H_{*}\left(B^{+}\right) .
$$

Since for all $t$, the maps $v_{t}^{+}$and $h_{t}^{+}$induce surjections on homology, it follows now from the surgery formula that the rank of $H F_{\text {red }}^{+}\left(S_{p / q}^{3}(K)\right)$ is at least $2 q$. For example, suppose that is a cycle in $A_{s}^{+}$representing a kernel of $v_{*}^{+}\left(\left[\xi_{s}\right]\right)$, we claim that $\left(s, \xi_{s}\right)$ can be completed to a cycle class in $\mathbb{X}_{i, p / q}^{+}$by adding terms of the form $\left(t, \xi_{t}\right) \in \mathbb{A}_{i}^{+}$and 
$\left(t, \eta_{t}\right) \in \mathbb{B}_{i}^{+}$with $t>s$. For example, since $v_{s+1}^{+}$induces a surjection on homology, we can find a cycle $\xi_{s+1} \in A_{\left\lfloor\frac{i+p(s+1)}{q}\right\rfloor}^{+}(K)$ and a chain $\eta_{s+1} \in B^{+}$with

$$
h_{s}^{+}\left(\xi_{s}\right)=v_{s+1}^{+}\left(\xi_{s+1}\right)+\partial \eta_{s+1}
$$

Proceeding inductively, we end up completing the initial cycle $\xi_{s}$ with a desired sequence of elements $\xi_{t} \in\left(t, A_{\left\lfloor\frac{i+p(s+1)}{q}\right\rfloor}^{+}(K)\right)$ and $\eta_{t} \in\left(t, B^{+}\right)$with $t \geq s$, so that

$$
h_{t}^{+}\left(\xi_{t}\right)=v_{t+1}^{+}\left(\xi_{t+1}\right)+\partial \eta_{t+1} .
$$

Moreover, for degree reasons, we can assume that the elements $\xi_{t}$ and $\eta_{t}$ vanish for sufficiently large $t$. Thus, the sum of these elements can be viewed as a homology class in $H_{*}\left(\mathbb{X}_{i, p / q}^{+}\right)$whose projection to $H_{*}\left(A_{\left\lfloor\frac{i+p s}{q}\right\rfloor}^{+}\right)$is the given kernel element $\left[\xi_{s}\right]$ initial kernel element. This proves that if for $s>0, H^{\text {red }}\left(A_{s}^{+}\right) \neq 0$, we construct elements in $\mathbb{X}_{i, p / q}^{+}$, one for for each time the chain complex $A_{s}^{+}$with appears ind $\mathbb{X}_{i, p / q}^{+}$, representing a homology class in $H_{*}\left(\mathbb{X}_{i, p / q}^{+}\right)$whose projection to $H_{*}\left(A_{s}^{+}\right)$has non-trivial image in $H^{\text {red }}\left(A_{s}^{+}\right)$. An analogous argument applies for the case where $s<0$. In view of this argument, the surgery formula now guarantees that $H F_{\text {red }}^{+}\left(S_{p / q}^{3}(K)\right)$ has rank at least $2 q$.

Suppose now that $H^{\text {red }}\left(A_{s}^{+}\right)=0$, but still the map induced by $v_{s}^{+}$is not an isomorphism. It follows at once that the rank of its kernel $n$ is positive. Indeed, for all $0 \leq t \leq s$, we see that $v_{t}^{+} A_{t}^{+} \longrightarrow B^{+}$factors through the natural projection from $A_{t}^{+}$ to $A_{s}^{+}$. It follows easily that the kernel of $v_{t}^{+}: H_{*}\left(A_{t}^{+}\right) \longrightarrow H_{*}\left(B^{+}\right)$for each such $t$ has dimension at least $m$.

Consider a $\operatorname{Spin}^{c}$ structure $i \in \mathbb{Z} / p \mathbb{Z}$ modeled on $\mathbb{X}_{p / q, i}^{+}(K)$. Let $k$ denote the number of copies of $A_{t}^{+}$with $|t| \leq s$ which appear in this model, and suppose that $k>0$. Then, it follows readily that $-\left(d\left(S_{p / q}^{3}(K), i\right)-d\left(S_{p / q}^{3}(O), i\right)\right) \geq 2 n$; and also that $\operatorname{rk} H F_{\text {red }}\left(S_{p / q}^{3}(K)\right) \geq n(k-1)$.

Thus, we see that

$$
\operatorname{rk} H F_{\text {red }}^{+}\left(S_{p / q}^{3}(K)\right)-\left(\frac{d\left(S_{p / q}^{3}(K), i\right)-d\left(S_{p / q}^{3}(O), i\right)}{2}\right) \geq q(2|s|+1) n \geq 2 q
$$

Lemma 3.3. Let $K \subset S^{3}$ be a knot with genus $g$. Then, there is a short exact sequence

$$
0 \longrightarrow \widehat{\operatorname{HFK}}(K, g) \longrightarrow H_{*}\left(A_{g-1}^{+}\right) \stackrel{v_{g-1}^{+}}{\longrightarrow} H_{*}\left(B^{+}\right) \longrightarrow 0
$$

Proof. There is an obvious short exact sequence

$$
0 \longrightarrow C\{(-1, g-1)\} \longrightarrow A_{g-1}^{+} \stackrel{v_{g-1}^{+}}{\longrightarrow} B^{+} \longrightarrow 0
$$


inducing a long exact sequence in homology. On the other hand, the map on homology $v_{g-1}^{+}$is surjective for simple algebraic reasons. (as it is an isomorphism in all sufficiently large degrees, it is $U$-equivariant, and the automorphism of $H_{*}\left(B^{+}\right)$induced by $U$ is surjective.) Finally, note that $H_{*}(C\{(-1, g-1)\}) \cong \widehat{\operatorname{HFK}}(K, g)$.

Proof of Theorems 1.1 and 1.2. By reflecting the knot if necessary, we can assume that $p / q>0$. Assume that $K$ is a knot with $S_{p / q}^{3}(K) \cong S_{p / q}^{3}\left(K_{0}\right)$, with $K_{0} \in\left\{T_{r}, T_{\ell}, S\right\}$. Combining Propositions 3.1 and 3.2, we conclude that for all $s>0, v_{s}^{+}: A^{+} \longrightarrow B^{+}$ induces an isomorphism on homology. From Lemma 3.3, we conclude that $\widehat{\operatorname{HFK}}(K, s)=$ 0 for all $s>1$. Note that this already proves that the genus of $K$ is one, cf. [7].

Now, we claim that

$q \cdot \operatorname{rkKer}\left(v^{+}: H_{*}\left(A_{0}^{+}(K)\right) \longrightarrow H_{*}\left(B^{+}\right)\right)=\operatorname{rk} H F_{\text {red }}^{+}(K)-\sum_{i}\left(\frac{d\left(S_{p / q}^{3}(K), i\right)-d\left(S_{p / q}^{3}(O), i\right)}{2}\right)$.

In view of Proposition 3.1, we conclude that $\operatorname{Ker}\left(v^{+}: H_{*}\left(A_{0}^{+}(K)\right) \longrightarrow H_{*}\left(B^{+}\right)\right)$has rank one. Thus, by Lemma 3.3, we see that $\widehat{\operatorname{HFK}}(K, 1)$ has rank one. By Ghiggini's theorem, it follows that $K$ is either the figure eight knot or the trefoil.

Another look at the Floer homology groups $S_{p / q}^{3}\left(K_{0}\right)$ as stated in Proposition 3.1 then allows one to conclude that $K=K_{0}$.

Proof of Corollary 1.3. Note that $\Sigma(2,3,7)$ cannot be realized as $1 / n$ surgery on any knot in $S^{3}$. This follows from the surgery formula for Casson's invariant $\lambda$, together with the fact that $|\lambda(\Sigma(2,3,7))|=1$. The corollary is now a direct application of Theorems 1.1 and 1.2. 


\section{REFERENCES}

[1] K. A. Frøyshov. The Seiberg-Witten equations and four-manifolds with boundary. Math. Res. Lett, 3:373-390, 1996.

[2] P. Ghiggini. Knot Floer homology detects genus-one fibred links. math.GT/0603445.

[3] P. B. Kronheimer, T. S. Mrowka, P. S. Ozsváth, and Z. Szabó. Monopoles and lens space surgeries. math.GT/0310164, to appear in Ann. of Math.

[4] P. S. Ozsváth and Z. Szabó. Holomorphic triangles and invariants for smooth four-manifolds. math.SG/0110169, to appear in Adv. in Mathematics, 2001.

[5] P. S. Ozsváth and Z. Szabó. Absolutely graded Floer homologies and intersection forms for fourmanifolds with boundary. Advances in Mathematics, 173(2):179-261, 2003.

[6] P. S. Ozsváth and Z. Szabó. Heegaard Floer homology and alternating knots. Geom. Topol., 7:225254, 2003.

[7] P. S. Ozsváth and Z. Szabó. Holomorphic disks and genus bounds. Geom. Topol., 8:311-334, 2004.

[8] P. S. Ozsváth and Z. Szabó. Holomorphic disks and knot invariants. Adv. Math., 186(1):58-116, 2004.

[9] P. S. Ozsváth and Z. Szabó. Holomorphic disks and topological invariants for closed threemanifolds. Ann. of Math. (2), 159(3):1027-1158, 2004.

[10] P. S. Ozsváth and Z. Szabó. Knot Floer homology and rational surgeries. math.GT/0504404, 2005.

[11] J. A. Rasmussen. Floer homologies of surgeries on two-bridge knots. Algebr. Geom. Topol., 2:757789, 2002.

[12] J. A. Rasmussen. Floer homology and knot complements. PhD thesis, Harvard University, 2003.

Department of Mathematics, Columbia University, New York, NY 10027

petero@math. columbia.edu

Department of Mathematics, Princeton University, New Jersey 08544

szabo@math.princeton. edu 